
\title{
Hydrofoils in Heaving Motion
}

Unsteady Force Measurements on Fully Wetted

\author{
by G. J. Klose ${ }^{1}$ and A. J. Acosta ${ }^{2}$
}

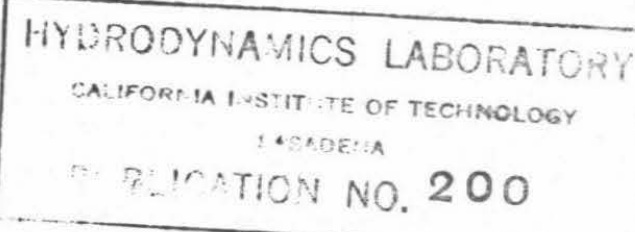

\begin{abstract}
An experimental investigation is reported of the unsteady forces due to heaving motion of fully wetted hydrofoils of unity aspect ratio and also in two-dimensional flow. The tests covered a broad range of reduced frequency and determined the effects of variation in submergence depth, angle of attack, oscillation amplitude, and flow velocity. In general, the findings agree well with available theoretical calculations, but some unexpected variations were found for the case of a wedge-shaped foil and for changes in angle of attack.
\end{abstract}

\section{Introduction}

KNOWLEDGE of the unsteady forces acting on an oscillating hydrofoil travelling beneath a free surface is necessary for the analysis of hydroelastic instability, such as flutter and divergence, and for dynamic response calculations. Extensive summaries of previous work in these fields are available elsewhere $[1,2,3]^{3}$ and need not be repeated here, but it is evident that many aspects of the unsteady hydrofoil problem still await successful consideration.

The separate effects of submergence depth, Froude number, and finite aspect ratio all contribute to the complexity of the theoretical solutions, and in many cases where a problem has in principle been solved, the compilation of numerical results has been discouraged by the prohibitive computational effort required. The extent of the experimental work published so far is also limited; for example, only one set of tests $[4,5]$ has been reported for the case of a hydrofoil oscillating under a free surface.

The objective of the present work was the experimental determination of the unsteady forces in heaving motion acting on a hydrofoil with unity aspect ratio and in twodimensional flow over a broad range of reduced frequencies with various values of submergence depth, angle of attack, oscillation amplitude, and flow velocity.

\section{Test Equipment and Procedure}

Water Tunnel

The experiments were carried out in the Free Surface Water Tunnel of the Hydrodynamics Laboratory at the California Institute of Technology, a detailed description of which is given in [6]. The working section is $20 \mathrm{in}$. wide and $8 \mathrm{ft}$ long; the normal depth of water is $20 \mathrm{in}$., with the free surface open to the atmosphere. Anskim-

\footnotetext{
${ }^{1}$ Now with Electro-Optical Systems, Pasadena, Calif.

${ }^{2}$ California Institute of Technology, Pasadena, Calif.

${ }^{3}$ Numbers in brackets designate References at end of paper.

Manuscript received at SNAME Headquarters, June 28, 1967.
}

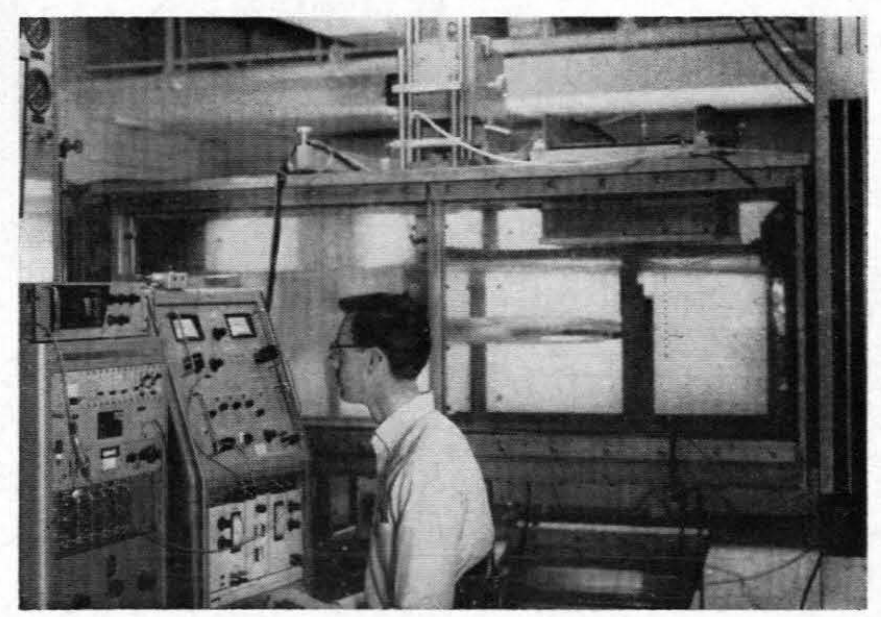

Fig. 1 View of working section and test equipment

mer located at the entrance to the working section removes the boundary layer built up along the top wall of the tunnel circuit, so the flow velocity in the working section is uniform right up to the free surface. The maximum flow velocity is about $27 \mathrm{fps}$. A pair of working section inserts was fabricated which split the flow into three streams, allowing the simulation of two-dimensional flow past the hydrofoils in the center stream. A view of the working section during a test run with the inserts installed is shown in Fig. 1.

\section{Hydraulic Oscillator}

The hydrofoil models are driven by a hydraulic oscillator, Fig. 2, which consists of a double-acting hydraulic piston with the admission of fluid regulated by an electronically controlled servovalve. The usable force output capability of the oscillator is about $300 \mathrm{lb}$ at a supply pressure of 5000 psi. With a set of mounting adapters, the oscillator can be mounted so as to place the hydrofoil submergence at any desired value. 


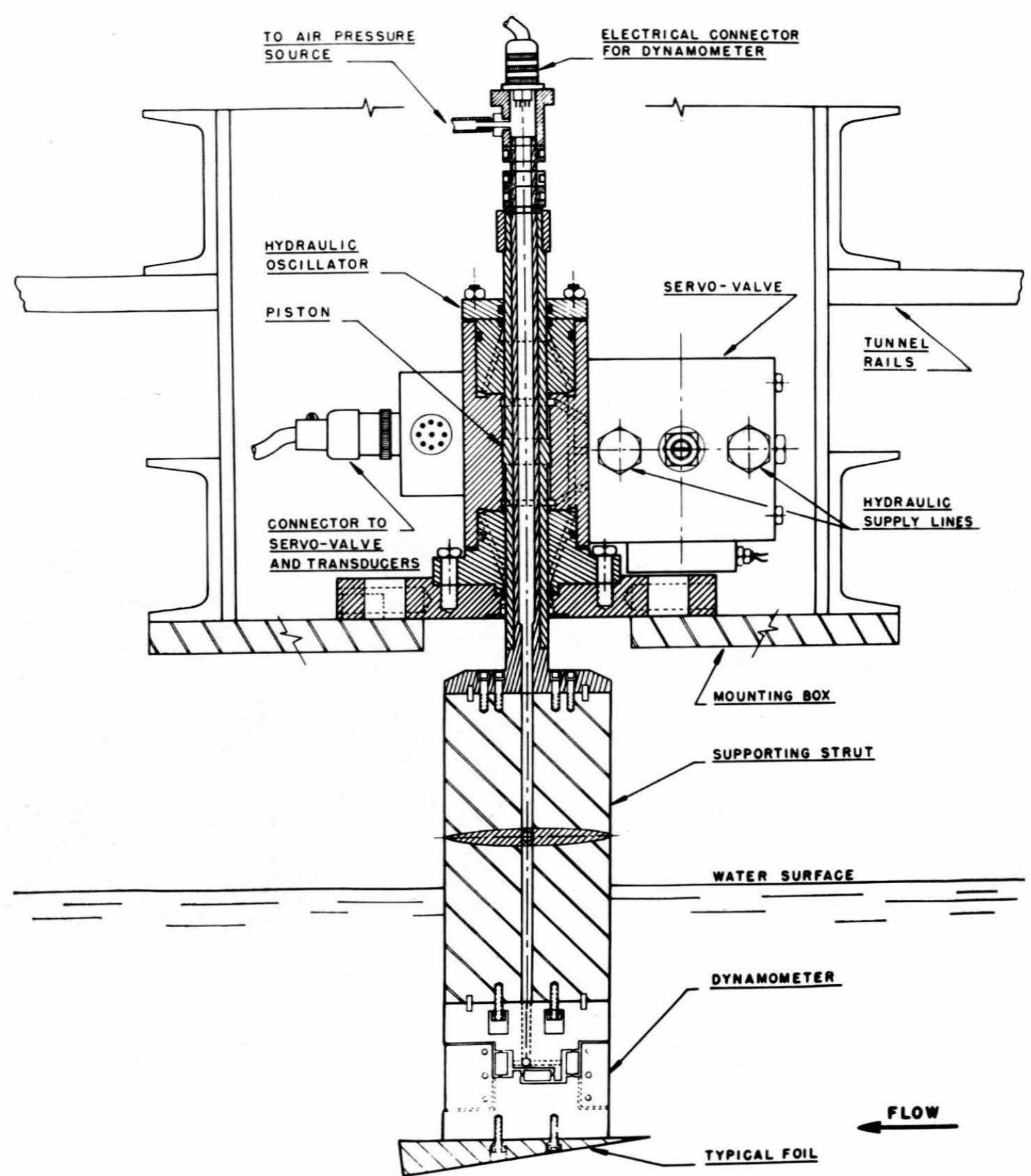

Fig. 2 Cross-section drawing of hydraulic oscillator. showing tunnel mounting, strut dynamometer, and hydrofoil

\section{Supporting Strut and Hydrofoil Models; Dynamometer}

The supporting strut is a symmetrical streamline section of NACA 0010 contour with 4 -in. chord, and is attached to the piston rod of the hydraulic oscillator. It is designed to maintain fully wetted flow at all times, so that ventilation from the free surface will not occur along its trailing edge. The hydrofoils are mounted to the bottom of the strut, the strut-foil combination thus forming an upside-down " $T$ ". The foils all have a 6-in. chord and 6.10-in. span, giving an aspect ratio of essentially unity. Foils were made with several cross-sectional shapes, including an 8-deg apex-angle wedge, a "flat plate" model of $1 / 4$-in. thickness with the leading and trailing edges smoothly tapered to a sharp edge (both these having square side edges), and a "rounded flat plate" which is $1 / 4$-in. thick like the "flat plate" but has the side edges rounded to a semicircle and the leading edge tapered down to a $1 / 16$-in. radius; the trailing edge 


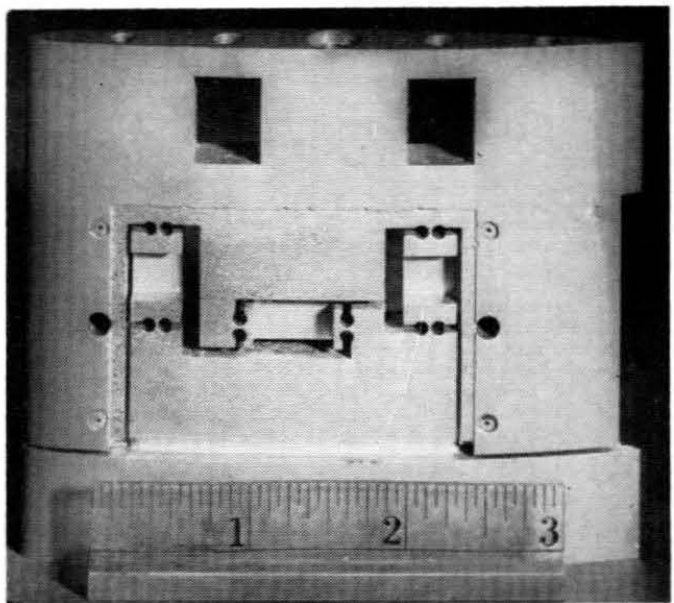

Fig. 3 Strut dynamometer before affixing strain gages

of this model is sharp like the "flat plate." Provision is made for mounting the foils at various static angles of attack.

The force dynamometer is constructed as part of the supporting strut, immediately above the hydrofoil. The general layout of the dynamometer can be seen in Fig. 3, which shows the flexures and the stressed elements before affixing the strain gages. There are three active elements, two in the lift direction and one in drag; the total lift force is obtained by adding the output of the two lift elements. Thin steel sheets are fitted in the recesses on each side of the dynamometer, and since it has to operate submerged, the entire section is waterproofed by cementing thin rubber sheet around the exterior. The completed dynamometer is very stiff in deflection and has no natural frequencies below $200 \mathrm{~Hz}$.

\section{Instrumentation}

The motion of the hydraulic oscillator is controlled by a servovalve, the electrical signals for which are generated by a servocontroller. The servocontroller incorporates a feedback loop which compares the output of a displacement or a velocity transducer to the command signal, thus forcing the hydrofoil to perform the desired motions. In practice, only sinusoidal excitations have been used. The command signal is generated by a low-frequency oscillator; this waveform also serves as the frequency and phase reference for the analysis of the output signals from the dynamometer and the transducers, which is performed by the Return Signal Analyzer. ${ }^{4}$ This unit carries out a Fourier analysis of the signal returning from the system under test and resolves it into an in-phase and a quadrature phase $(90 \mathrm{deg}$ ) component relative to the reference signal. Only that part of the signal which is of the same frequency as the reference waveform is detected; all other frequencies, and noise, are eliminated with a rejection specified at $40 \mathrm{db}$. Since the funda-

${ }^{4}$ Boonshaft and Fuchs, Inc., Hatboro, Pa.

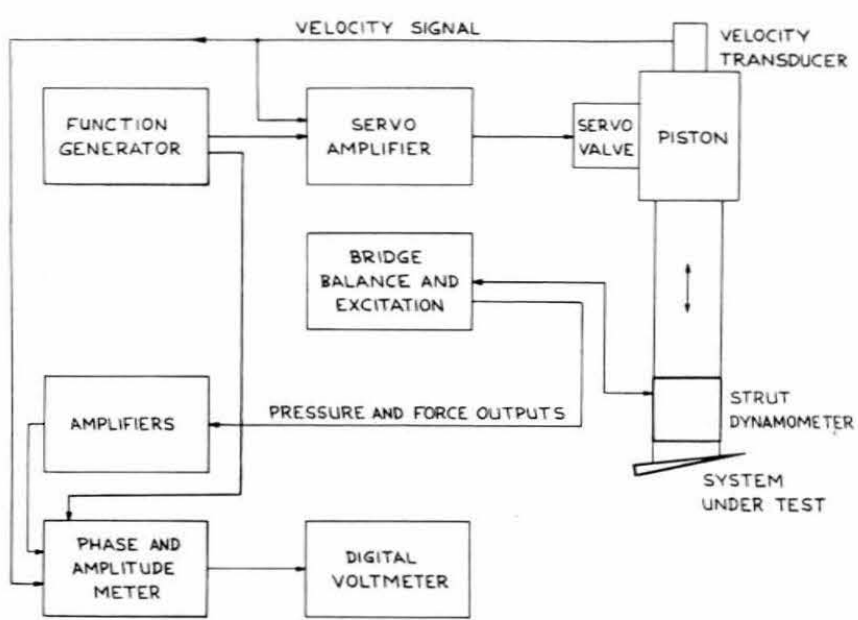

Fig. 4 Diagram of electronic equipment for hydraulic oscillator and data acquisition

mental frequency component of the signal is of primary interest, the loss, with the present apparatus, of all information about harmonics and other frequencies is not an important drawback. The rms amplitudes of the in-phase and quadrature components are given by the Return Signal Analyzer as dc outputs; these are read on a time-averaging digital voltmeter.

\section{Calibrations}

Accurate measurement of the oscillation amplitude is essential for determination of the dynamic forces in experiments such as the present ones. The hydraulic oscillator incorporates both a position and a velocity transducer. The position transducer was calibrated statically by measuring displacement with a travelling microscope. Two different means were used to calibrate the velocity transducer. Its output was recorded on an oscillograph together with that of the calibrated position transducer to establish the velocity calibration. The same signals were also processed through the Return Signal Analyzer, thus verifying the calibration of that instrument. A somewhat different way of calibrating the velocity transducer is simply to compare the fundamental components of the two transducer outputs, as measured by the Return Signal Analyzer. In either case, the effects of imperfections in the output waveform of the hydraulic oscillator on these calibrations are avoided. Separate measurements of the total harmonic distortion in the oscillation velocity waveform showed this to be below five percent in all cases, most of which was third harmonic.

To ascertain proper functioning of the strut dynamometer, the output of each of the force elements was measured statically for various combinations of lift, pitching moment, and drag. Lift output was free of interactions from pitching moment, drag, and roll moment, and an equivalent result was found for pitching moment. Drag, however, showed an unacceptable output due to roll moment and was not recorded in the present experiments. 
For the actual data runs, dynamic calibrations using a known mass were performed and repeatedly checked.

\section{Test Parameters}

As it was not possible to carry out tests with every model over the whole matrix of variables desired, a scheme was evolved of essentially picking one configuration as a standard and varying one parameter at a time. The oscillation frequencies selected were $3,5,8,10,15$, 20,30 , and $40 \mathrm{cps}$; this gave reduced frequencies ranging from 0.43 to 5.17 at the normal flow velocity of $11 \mathrm{fps}$ and from 0.29 to 3.81 at $16.5 \mathrm{fps}$. The investigation of even lower values of reduced frequency was prevented by the low-frequency limitations of the Return Signal Analyzer used on the one hand and mechanical damage to the waterproofing at higher flow velocities on the other. Submergences with the aspect-ratio-one foils were 0.25 , 0.5 , and 1.7 times the hydrofoil chord (6 in.), and 1.2 chords for two-dimensional flow using the test section inserts. The static angle of attack could be varied from $-12 \mathrm{deg}$ to $+12 \mathrm{deg}$. The oscillating angle of attack due to the hydrofoil motion was nominally 4 deg at most frequencies, and some runs were made with 50 percent and 150 percent of this amplitude.

\section{Data Reduction}

The measured quadrature lift forces were corrected for the inertial force due to the strut and hydrofoil mass; this was calculated for each data point using the measured oscillation amplitude. Calculation was considered both more straightforward and reliable than the method sometimes used of injecting a signal proportional to the correction term into the electrical signal generated by the force dynamometer.

The contribution to the measured lift of the forces on the small section of strut between the force dynamometer and the hydrofoil is judged to be negligible, and thus no attempt at a correction was made. The effect of the disturbance to the flow about the hydrofoil caused by the presence of the strut could only be measured by devising an entirely different mounting and oscillating system, which was not feasible here; since in fact hydrofoils are frequently supported by just such a strut, the present measurements are quite pertinent, and in any event the

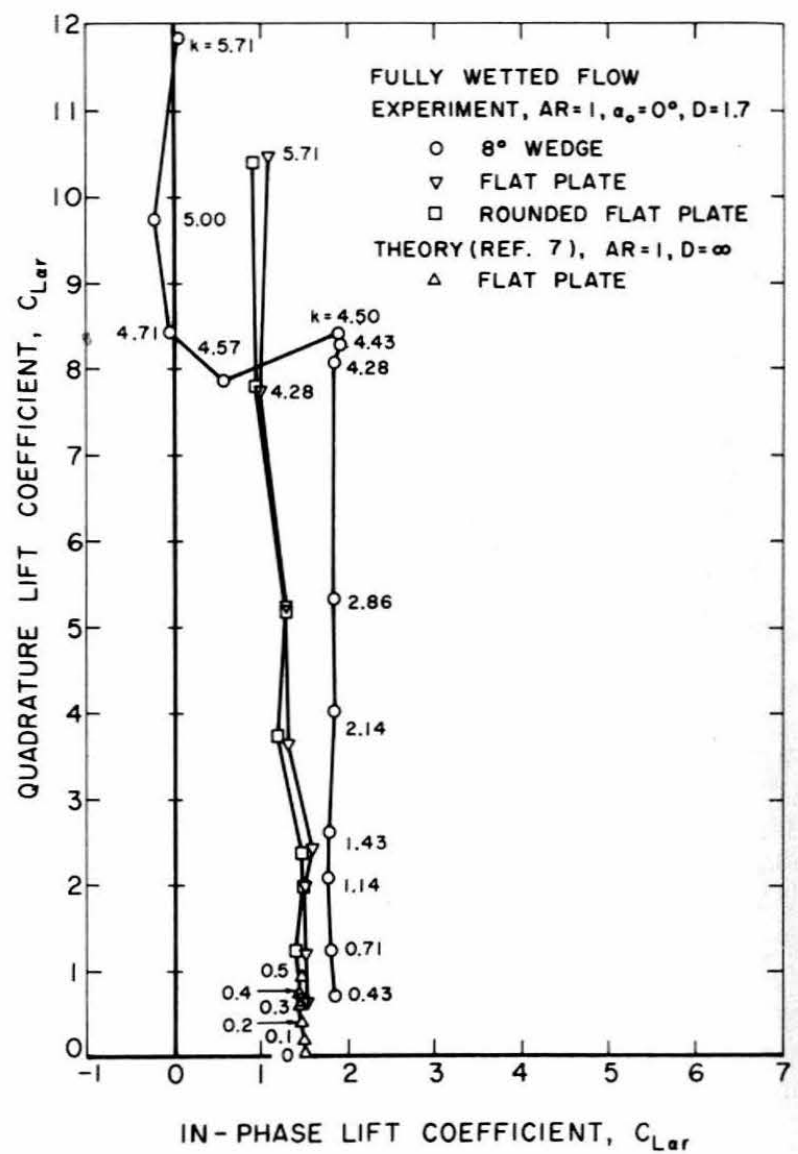

Fig. 5 Variation of unsteady lift coefficients with reduced frequency for several aspect-ratio-one foils in fully wetted flow at 1.7 chords submergence

effect should not be large. Also, no corrections have been made for tunnel interference or blockage, which are expected to be small in these free-surface flows.

\section{Discussion of Results}

\section{Aspect Ratio One}

Representative Results. Some introductory results, along with points calculated from Laidlaw's theory [7],

Nomenclature

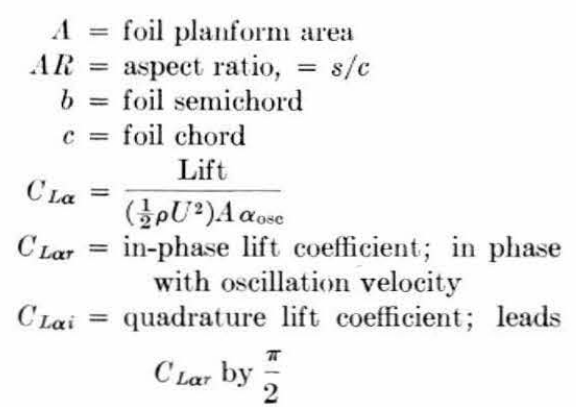

$$
\begin{aligned}
& D=\begin{array}{c}
\text { submergence depth in terms of } \\
\text { hydrofoil chord }
\end{array} \\
& F=\text { Froude number based on semi- } \\
& \qquad \text { chord }=\frac{U}{\sqrt{ } b g} \\
& f=\text { frequency, eps, }=\omega / 2 \pi \\
& g=\text { acceleration due to gravity } \\
& k=\text { reduced frequency }=\frac{\omega b}{U}=\frac{\omega c}{2 U} \\
& s=\text { hydrofoil span }
\end{aligned}
$$

$$
\begin{aligned}
S t= & \text { Strouhal number }=f w / U \\
U= & \text { tunnel velocity } \\
V_{\text {ose }}= & \text { transverse velocity amplitude due } \\
& \quad \text { to heaving oscillation } \\
w= & \text { base height of wedge } \\
\alpha_{0}= & \text { stationary angle of attack } \\
\alpha_{\text {osc }}= & V_{\text {osc }} / U \\
\rho= & \text { density of water } \\
\phi_{L}= & \text { phase angle, } \tan ^{-1}\left(C_{L \alpha i} / C_{L \alpha r}\right) \\
\omega & =\text { frequency, radians per second }
\end{aligned}
$$



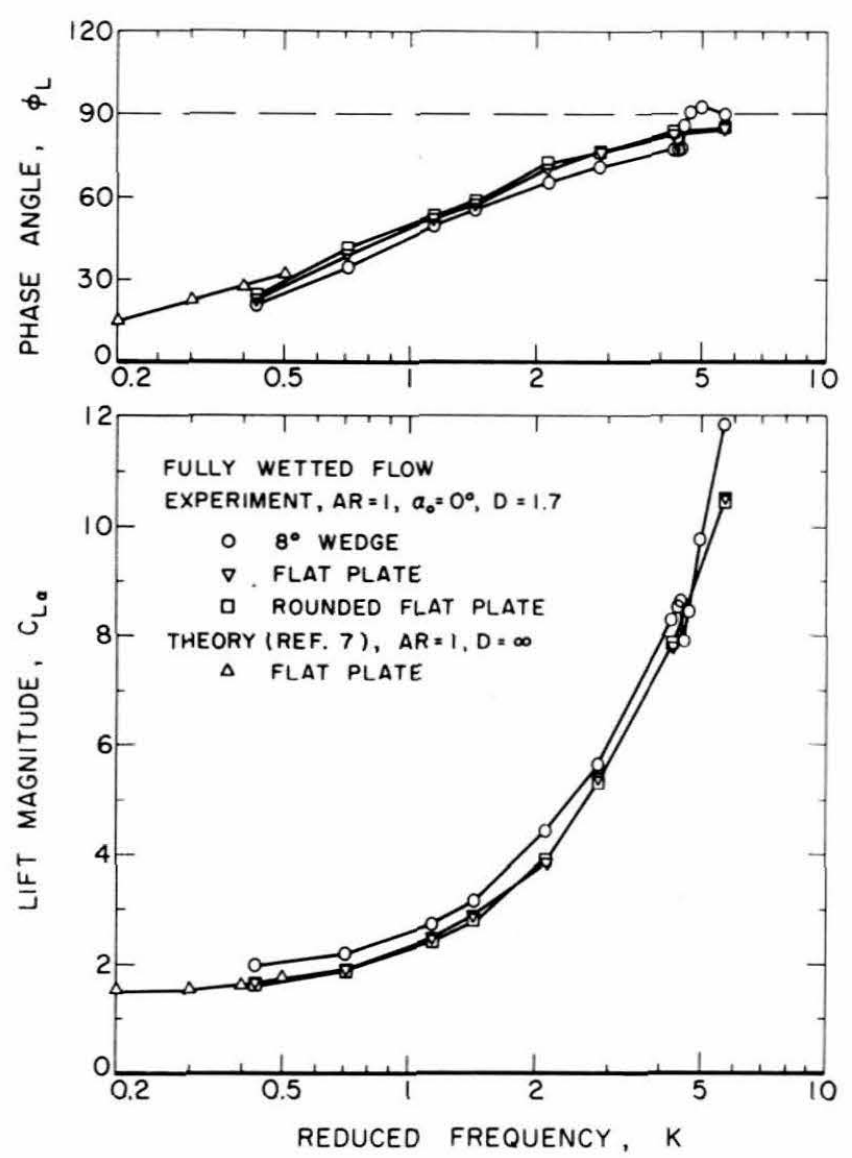

Fig. 6 Magnitude-and-phase plot of Fig. 5 data

are presented in Figs. 5 and 6 . These theoretical points are for infinite submergence, but comparing them with the experimental values taken at 1.7 chord lengths submergence should be reasonable since the surface effect is expected to have greatly diminished at this depth [12].

While the flat-plate theory is only available for reduced frequencies up to 0.5 , it is evident that the calculated quadrature lift is a little higher than the experimental values, due to a somewhat larger lift phase angle; on the whole, the agreement is remarkably good. The "rounded flat plate" has generally slightly lower values of in-phase lift coefficient than the "flat plate," which is reasonable since the rounded side edges give this model a slightly lower effective aspect ratio than the square-edged flat plate foil.

The values of the in-phase lift coefficient for the wedge foil are consistently larger than for the flat plate. This finding was unexpected, since the wedge is essentially just a flat plate with the thickness uniformly increasing toward the trailing edge. Unfortunately neither theoretical nor other experimental results are available for such a shape. In steady flow, airfoil theory predicts lift increasing with thickness [8], but in free-surface flows there is an appreciable decrease of lift due to thickness [9]. For unsteady flow, Wood's theory [10] gives a small

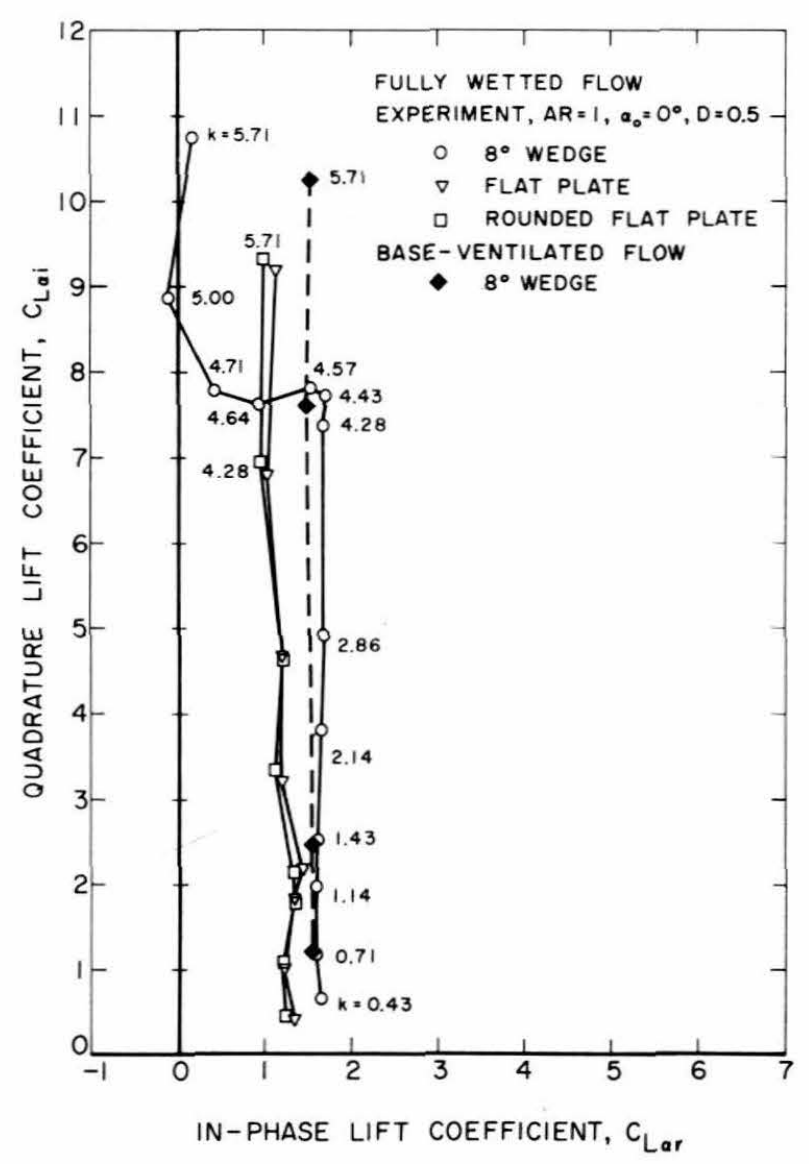

Fig. 7 Variation of unsteady lift coefficients with reduced frequency for several aspect-ratio-one foils in fully wetted flow and for the wedge with base-vented flow at 0.5 chords submergence

decrease in the in-phase lift coefficient due to thickness when viscosity is neglected. However, all these theories assume an airfoil-shaped section with a sharp trailing edge and are thus not really applicable to the wedge foil, so that the high in-phase lift of the wedge remains unexplained at present.

Results corresponding to Fig. 5 are shown for the other values of submergence in Figs. 7 and 8 . Comparison of these curves shows that, although the numerical values of the lift coefficients change with submergence, the shapes of the trajectories for the various foils are notably similar at each depth.

The most striking feature of the graphs is the behavior for the wedge foil at a reduced frequency of about 4.5. This "knee" in the curve has good reproducibility, Fig. 10, and appears to be a real physical effect, as will be discussed shortly.

Submergence Effect. The effect of varying submergence on the measured unsteady lift is shown in Fig. 9 for the flat-plate foil and in Fig. 10 for the wedge. Both the in-phase and the quadrature lift coefficient decrease as the foil approaches the surface. This seems to hold at all values of reduced frequency, except of course above the knee of the wedge curves. 


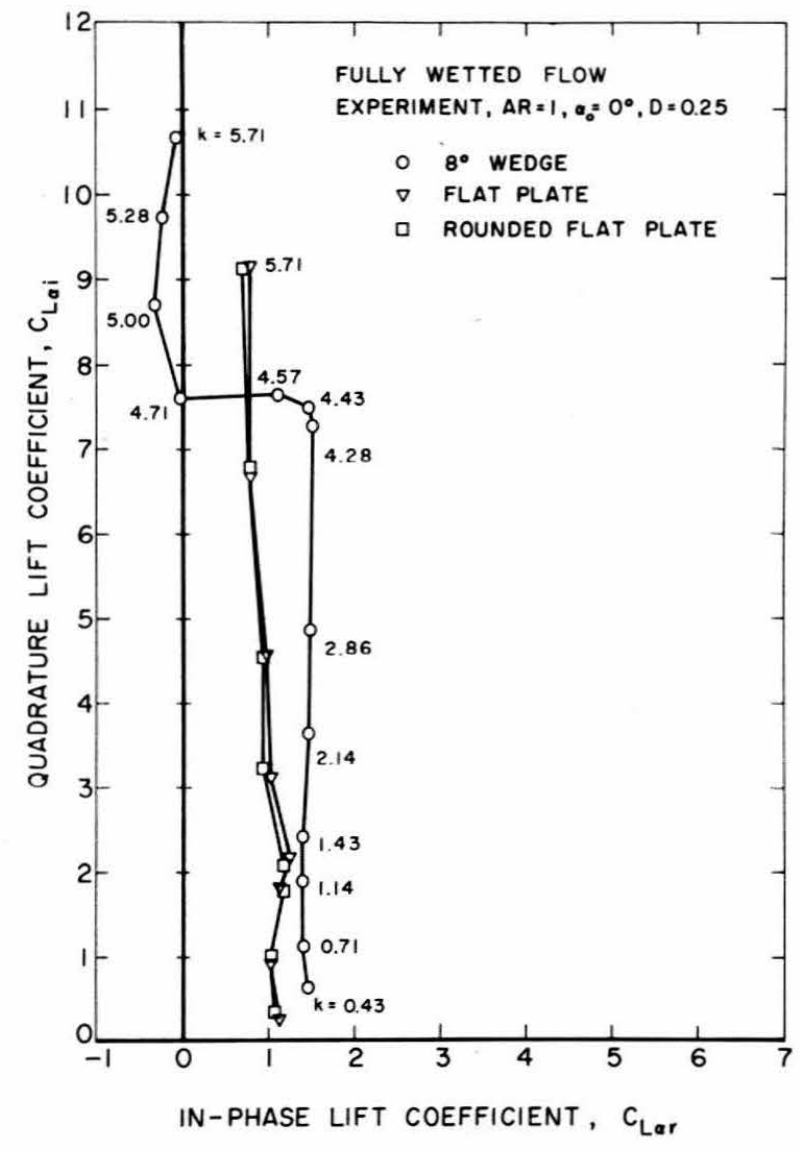

Fig. 8 Variation of unsteady lift coefficients with reduced frequency for several aspect-ratio-one foils in fully wetted flow at 0.25 chords submergence

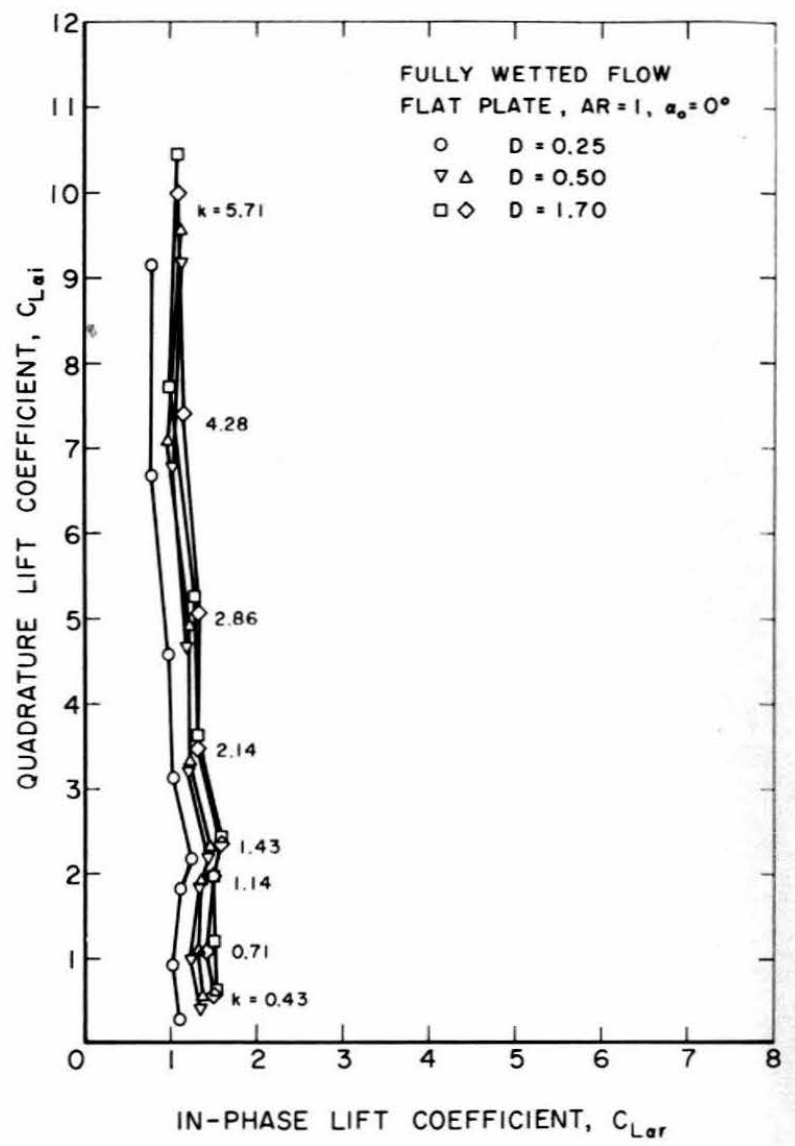

Fig. 9 Variation of unsteady lift coefficients with reduced frequency for the aspect-ratio-one flat plate in fully wetted flow, showing effect of submergence
At the present time, no numerical theoretical results exist for the effect of submergence depth on the unsteady lift of a finite-aspect-ratio foil. For infinite aspect ratio (two-dimensional flow), the theory of Crimi and Statler [11], which is plotted in Fig. 18, shows the same trend of submergence effect for reduced frequencies above about 1.0 as has been observed in the present experiments. This is not the case, however, for reduced frequencies below 0.6 , although this may not be significant because of the different aspect ratio.

Some experiments were also performed with the foils positioned quite close to the surface. It was found that fully wetted flow could be maintained over the flat-plate foil for submergences as small as 0.01 chords, which is $1 / 16$ in., with the foil at $+4 \mathrm{deg}$ angle of attack and oscillations of very small amplitude; and at 0.04 chords submergence, $1 / 4 \mathrm{in}$., the full range of reduced frequencies could be covered. The results of these tests are shown in Fig. 11, with the curve for 0.25 chords submergence included for comparison, and also some data that were taken with atmospheric ventilation of the upper surface (planing). Inspection of the graph shows that the quadrature lift decreases by about 25 percent as the surface is approached from 0.25 chords submergence, but the in-phase lift is decreased only slightly. For the planing foil, both components of the unsteady lift are reduced compared to the fully wetted foil at the same submergence, except that the quadrature lift is abnormally high for reduced frequencies below 1.0. In general, however, the lift values for fully wetted and ventilated flow approach each other as the surface is approached, as would be expected.

Reduced Frequency. To confirm the assumption that the reduced frequency is in fact the pertinent parameter in these tests, some runs were carried out with the freestream velocity increased by 50 percent to 16.5 fps. Recalling the definition of reduced frequency, it is seen that the same value of reduced frequency corresponds now to a 50-percent higher value of oscillation frequency. Fig. 12 shows the results of this check. While the highest frequency is now no longer large enough to reach the "knee," the curves show only minute differences in their common range. By increasing the oscillation frequency beyond the usual range in another set of tests (not shown here), it was established that with the higher flow velocity the "knee" occurs at the same reduced frequency as with the normal flow velocity.

Oscillation Amplitude. To ascertain that the more-or- 


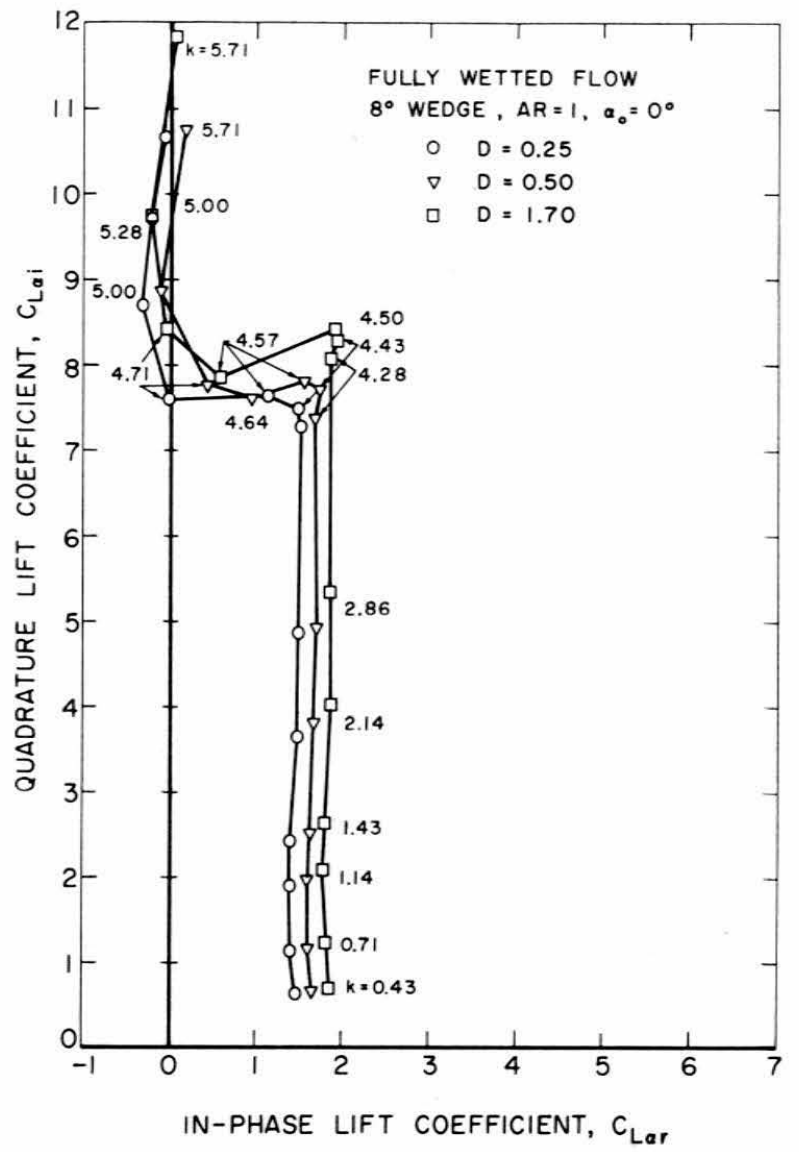

Fig. 10 Variation of unsteady lift coefficients with reduced frequency for aspect-ratio-one wedge in fully wetted flow, showing effect of submergence

less arbitrarily chosen oscillation amplitude does not affect the findings in some unsuspected way, a series of tests was performed in which only the oscillation amplitude was varied. Values of 50,100, and 150 percent of the usual excitation voltage were chosen; the resulting oscillation amplitudes are 2,4 , and $6 \mathrm{deg}$. These tests were performed with the wedge foil and the results are shown in Fig. 13. Although the differences in the coefficients are very small below the knee, both components of the unsteady lift seem consistently higher for greater oscillation amplitude.

Angle of Attack. It was found that the angle of attack $\alpha_{0}$ at which the foil is mounted on the strut has a significant effect on the in-phase lift coefficient for all the foils. This is shown for the flat-plate foil in Figs. 14 and 15; similar results were found also for the wedge foils.

For the flat plate, the curves for a given $\alpha_{0}$ are almost identical whether the angle is positive or negative (comparison of the two figures is facilitated by noting that the curve for $\alpha_{0}=0 \mathrm{deg}$ is common to both plots). There are no systematic variations with angle of attack in the quadrature component, although the close similarity of corresponding points on the positive and negative angleof-attack curves is noteworthy.

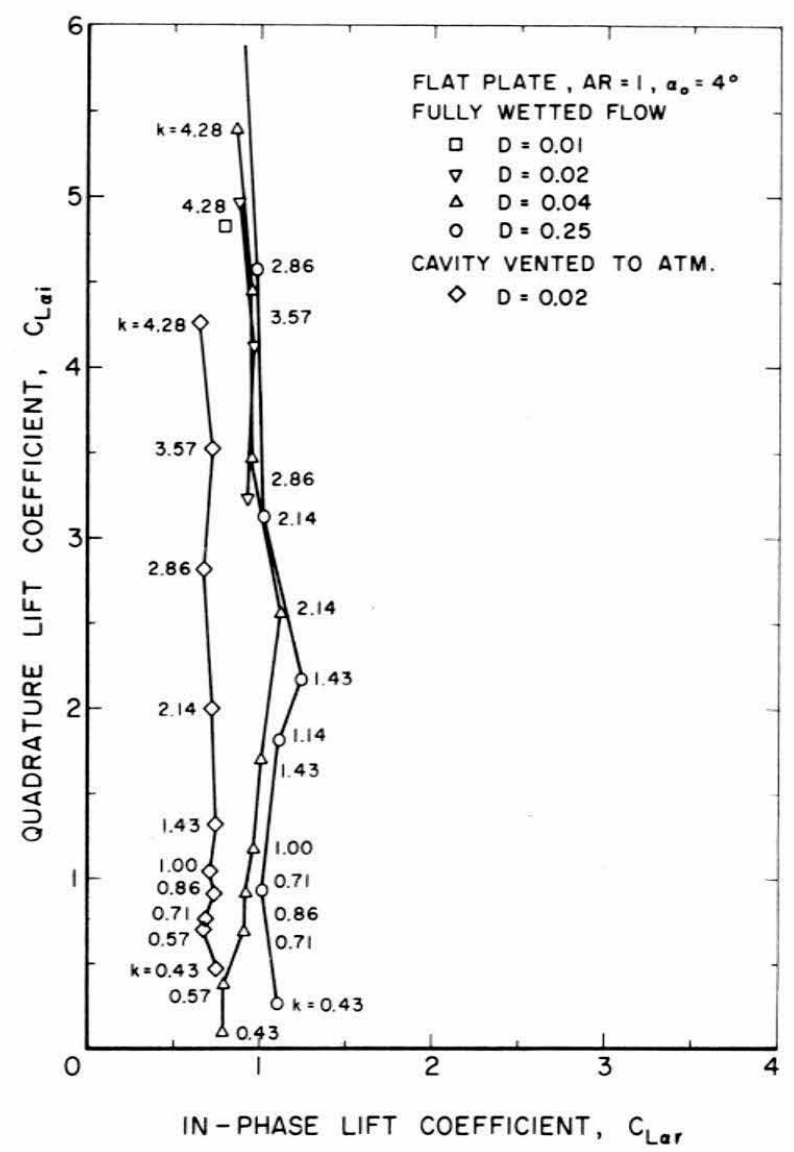

Fig. 11 Variation of unsteady lift coefficients with reduced frequency for aspect-ratio-one flat plate near free surface, showing effect of submergence

The same trend of lift increasing with angle of attack also seems to occur in two-dimensional flow, judging by the results in reference [5]. These coefficients have been put into the form used here, and plotted in Fig. 16. These data are the preliminary revised results of reference [4]; while a considerable amount of scatter is evident, the points at the larger angle of attack appear to have the greater in-phase lift coefficient.

A likely reason for the increase of in-phase lift with angle of attack may be found in the nonlinearity of the lift-curve slope of low-aspect-ratio foils in steady flow [12]. At a higher set angle of attack $\alpha_{0}$, the oscillations take place on a steeper part of the lift-versus-angle of attack curve, and thus the measured fluctuating lift coefficient is larger.

Wedge Foil. As mentioned, the peculiar trajectory of the lift curve for the wedge in the region of reduced frequency of 4.5 is a feature which persists for all the changes in experimental parameters. At a certain fairly well reproducible reduced frequency, the in-phase component decreases abruptly to near zero and in some cases even becomes negative; at the same point the rate of increase of quadrature lift with frequency becomes temporarily 


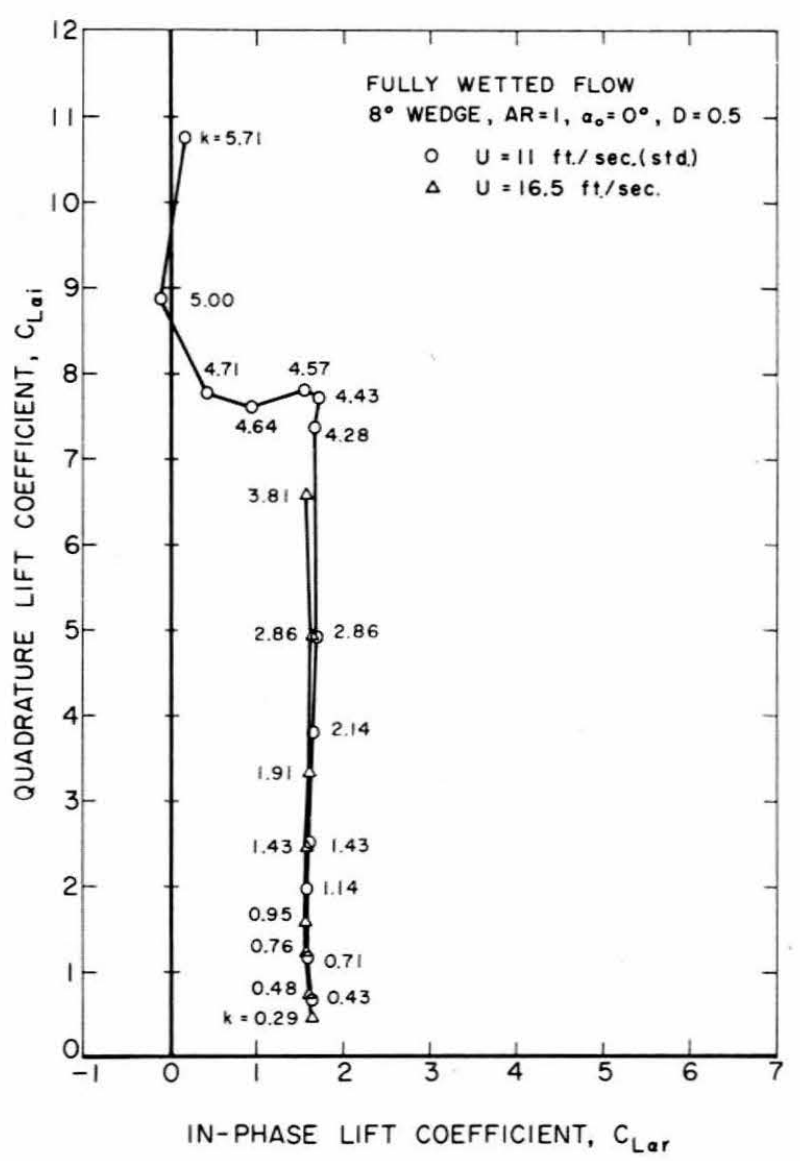

Fig. 12 Variation of unsteady lift coefficients with reduced frequency for aspect-ratio-one wedge in fully wetted flow, showing effect of flow velocity

negative and then rises somewhat irregularly as the reduced frequency is increased further.

It is physically reasonable to ascribe this phenomenon to the vortex shedding associated with the blunt base of the wedge. The Strouhal number $f w / U$ corresponding to the knee of the curve is about 0.21 based on the base height of the wedge. Previous experimental investigations by Young and Holl [13] of the wake behind stationary wedges gave a value of 0.2 to 0.25 for the Strouhal number based on the observed vortex shedding frequency; and studies by Bishop and Hassan [14] of the wake behind both stationary and oscillating cylinders transverse to the flow also gave Strouhal numbers around 0.20 . These latter tests were carried out at Reynolds numbers from 4000 to 11,000 , while Young and Holl had values around 200,000 based on chord length. Considering the wide range of Reynolds number covered by those investigations, it may reasonably be assumed that the same physical process occurs in the present tests which have a Reynolds number of 500,000 or, at the higher flow velocity, 750,000 .

Bishop and Hassan [14] also performed tests with cylinders oscillated in a range of frequencies bracketing the observed natural shedding frequency, in which vari-

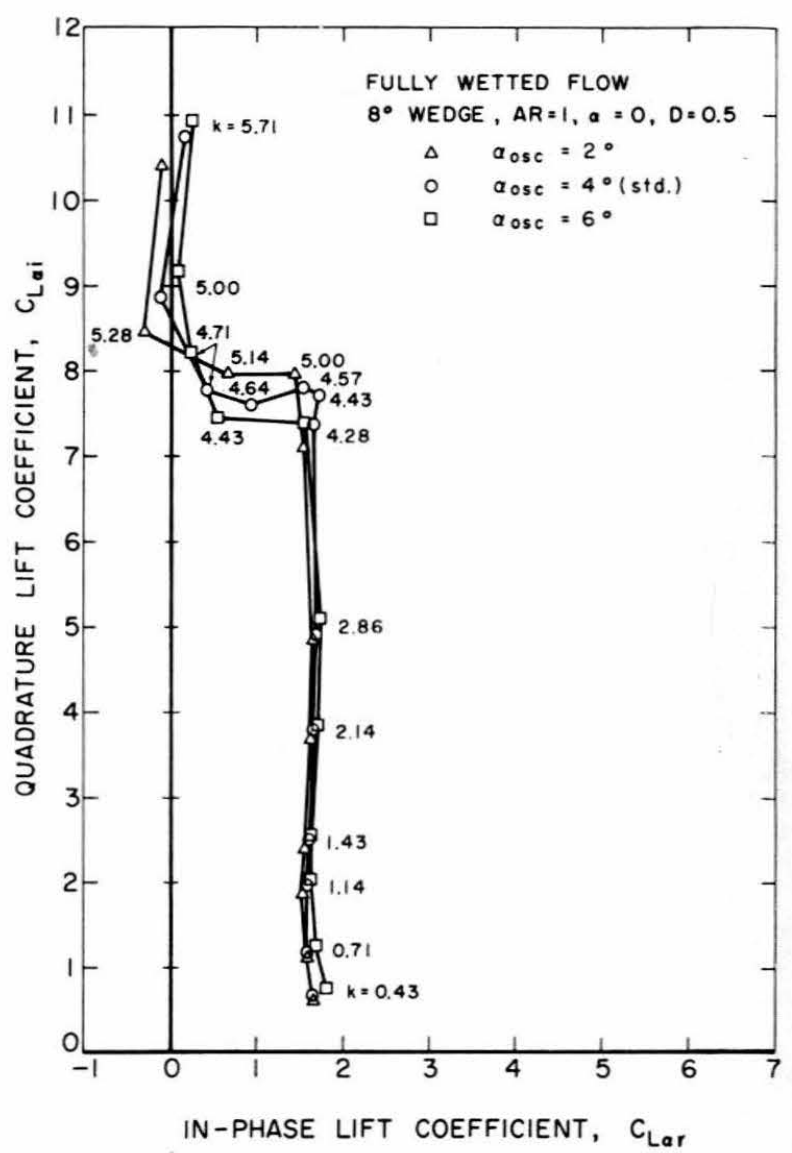

Fig. 13 Variation of unsteady lift coefficients with reduced frequency for aspect-ratio-one wedge in fully wetted flow, showing effect of oscillation amplitude

ous coupling effects between the frequency of the imposed motion and the natural frequency of the wake were observed, such as synchronization, frequency demultiplication, and beating. Based on these findings, they postulate the existence of a nonlinear self-excited "wake oscillator," analogous to a mechanical oscillator. Their observations certainly resemble the behavior found in the present tests, and the knee in the lift curves for the fully wetted wedge may justifiably be ascribed to such a phenomenon.

It is worthwhile to note at this point that when the wedge is base-ventilated so that the fluid from the two faces of the wedge can no longer interact in the near wake, the knee vanishes completely, as would be expected.

In an attempt to assess quantitatively the effect of the wake vortex shedding, the part of the lift coefficient trajectory below the knee was extrapolated to the higher values of reduced frequency by assuming the same trend for the curve as for the flat plate, i.e., neglecting the knee entirely. The vector difference between this extrapolated curve and the experimental points may thus be taken as a measure of the "wake vortex effect." This has been done for various flow configurations and the results 


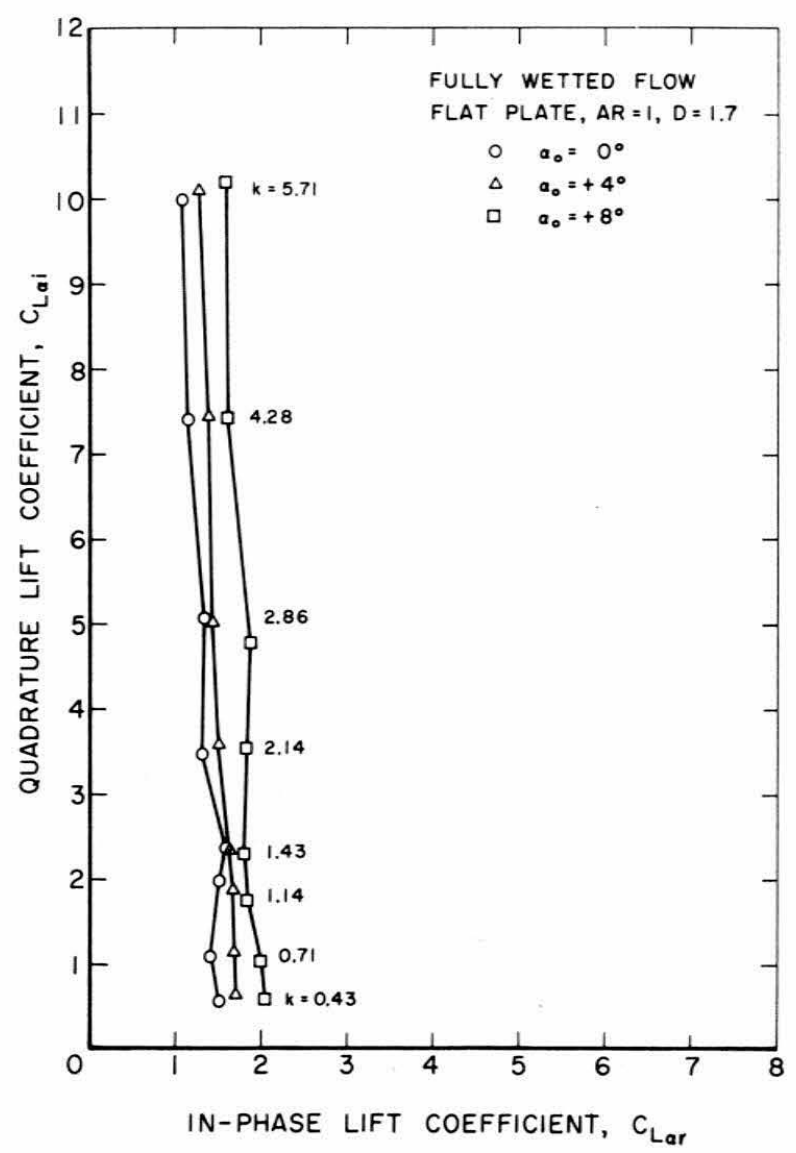

Fig. 14 Variation of unsteady lift coefficients with reduced frequency for aspect-ratio-one flat plate in fully wetted flow, showing effect of angle of attack

are plotted in Fig. 17, where the magnitude and phase of the difference in lift coefficient have been plotted against the Strouhal number based on wedge base height and oscillation frequency.

The lower-frequency limit of the wake effect seems fairly sharply defined, yet the range over which the wake effect is obtained is very broad. Similar behavior was found in the experiments of Eagleson et al. [15]. Unfortunately, it was not possible in the present test program to obtain data for even higher Strouhal numbers. It is reasonable to assume that decoupling between the forced foil oscillation and the wake effect will occur at some higher frequency, so that the experimental points would again coincide with the extrapolated curve.

Looking at Fig. 17 in detail, it is seen that the magnitude of the lift coefficient difference rises rapidly to about 1.9 for all the aspect-ratio-one foils and stays constant with increasing frequency; for two-dimensional flow, the magnitude is nearly twice as high. There is little change in the minimum Strouhal number with submergence, but a notable change with oscillation amplitude. The wake vortex effect at different oscillation amplitudes is appreciably better correlated when the total displacement

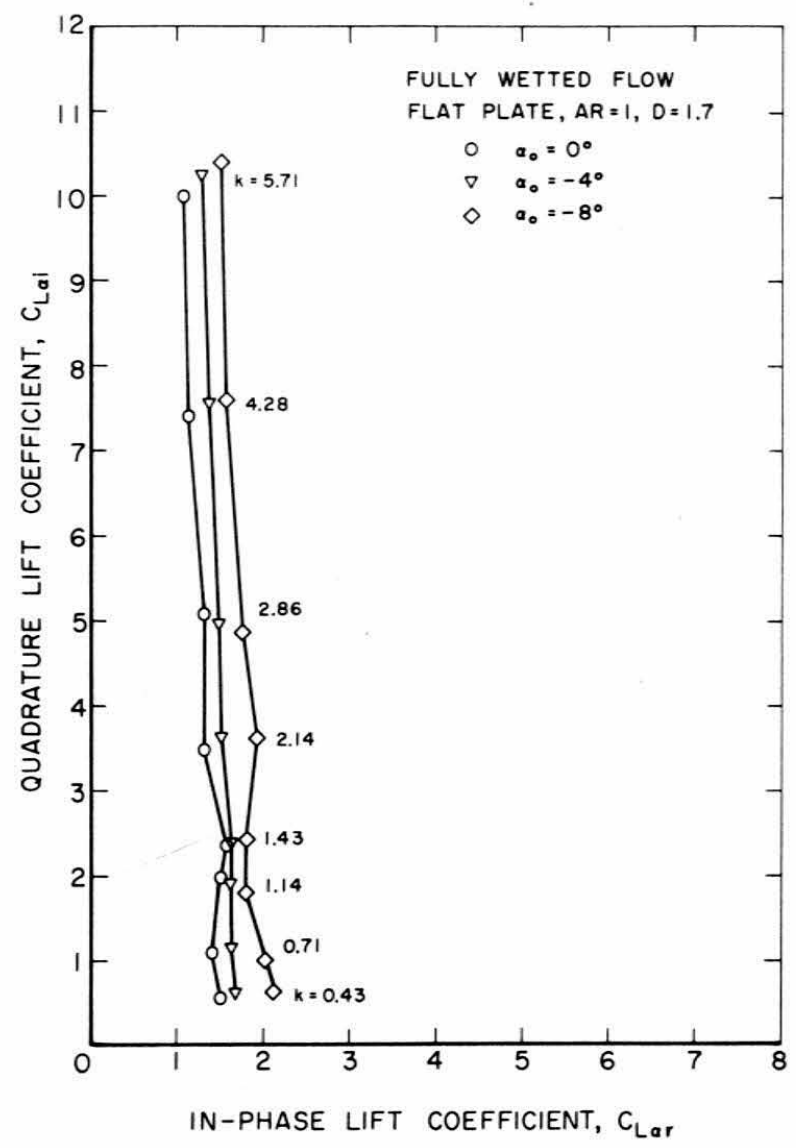

Fig. 15 Variation of unsteady lift coefficients with reduced frequency for aspect-ratio-one flat plate in fully wetted flow, showing effect of angle of attack

of the moving foil is added to the base height of the wedge in computing the Strouhal number.

No other published results for a similar configuration have been found; however, in reference [15] vortexforcing moments were calculated from observations of two-dimensional foils free to pitch about the leading edge. These coefficients are not directly comparable with the present force measurements, but their amplitude is of the same order of magnitude. Those tests gave no information about phase angles.

In the present tests, the phase angle of the vortex effect measured in the same sense as for the total lift reaches remarkably similar values for all test conditions at the higher Strouhal numbers. Near the minimum Strouhal number, there are some differences which appear to be connected mostly with the differences in oscillation amplitude. The values obtained with twodimensional flow are in the same range as those for aspectratio-one foils.

\section{Two-Dimensional Tests}

Tests with two-dimensional flow were performed as already mentioned at only one value of submergence. The theoretical effect of varying the submergence of an 


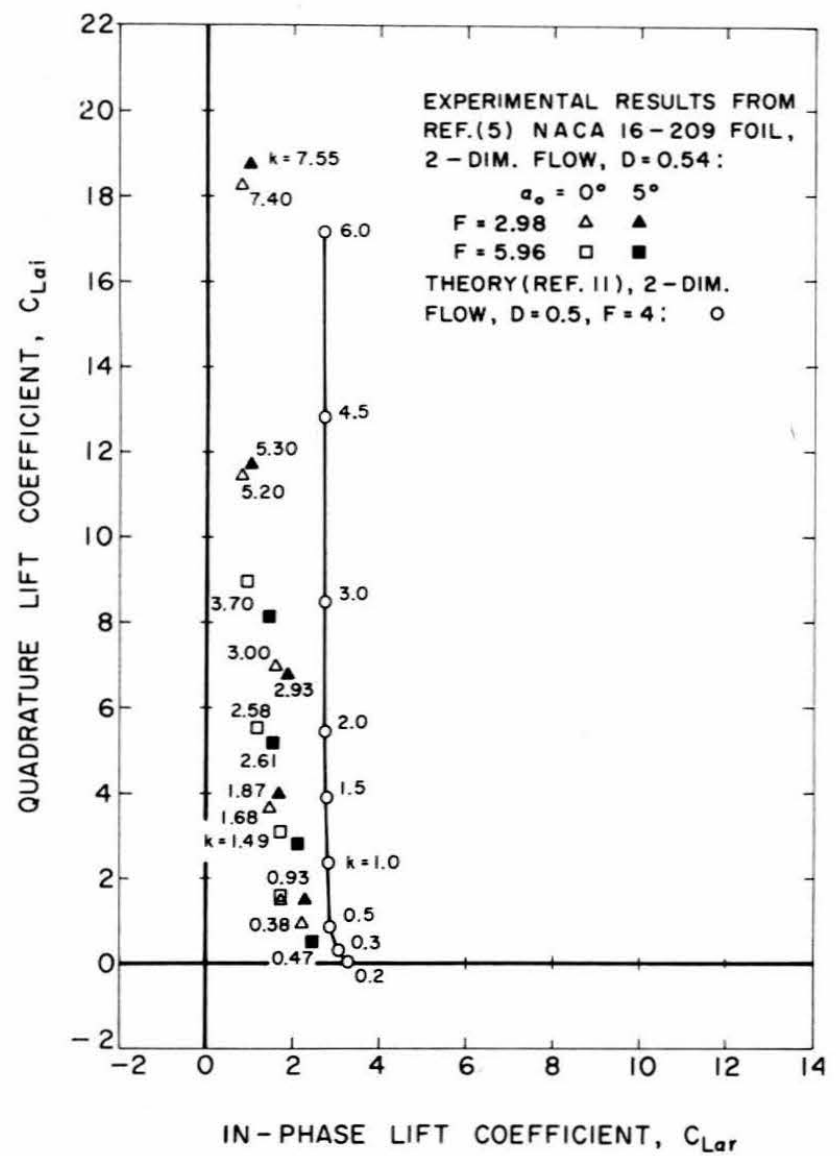

Fig. 16 Reference [5] data compared with theory of reference [11] for fully wetted two-dimensional hydrofoils

oscillating hydrofoil is shown for reference in the curves of Fig. 18 taken from the results of Crimi and Statler [11].

Representative Results. Some experimental results are shown in Fig. 19, along with the theoretical curve computed for the same value of reduced frequency from reference [11]. The experimental points are nearly equivalent to those of the theory; in the present tests the submergence is 1.2 chords and the Froude number is 3.88. The theoretical calculations used are for a submergence of 1.0 chords and Froude number of 4.0.

As in the case of the aspect-ratio-one foils, the in-phase lift of the wedge is again substantially larger than that of the flat plate and its quadrature lift is also somewhat larger, though this is not easily seen from Fig. 19. The theoretical results do not bear quite the same relationship to the experimental findings as in the aspect-ratio-one tests, where the flat plate had nearly the same lift magnitude as calculated. Here the lift magnitude of the flat plate is considerably lower than the calculated curve, and that of the wedge is also slightly lower than calculated. The phase angle is about $5 \mathrm{deg}$ lower for both foils than the theoretical values.

Smith and Sevik [17] in experiments with an airfoil oscillating in pitch found a similar discrepancy and
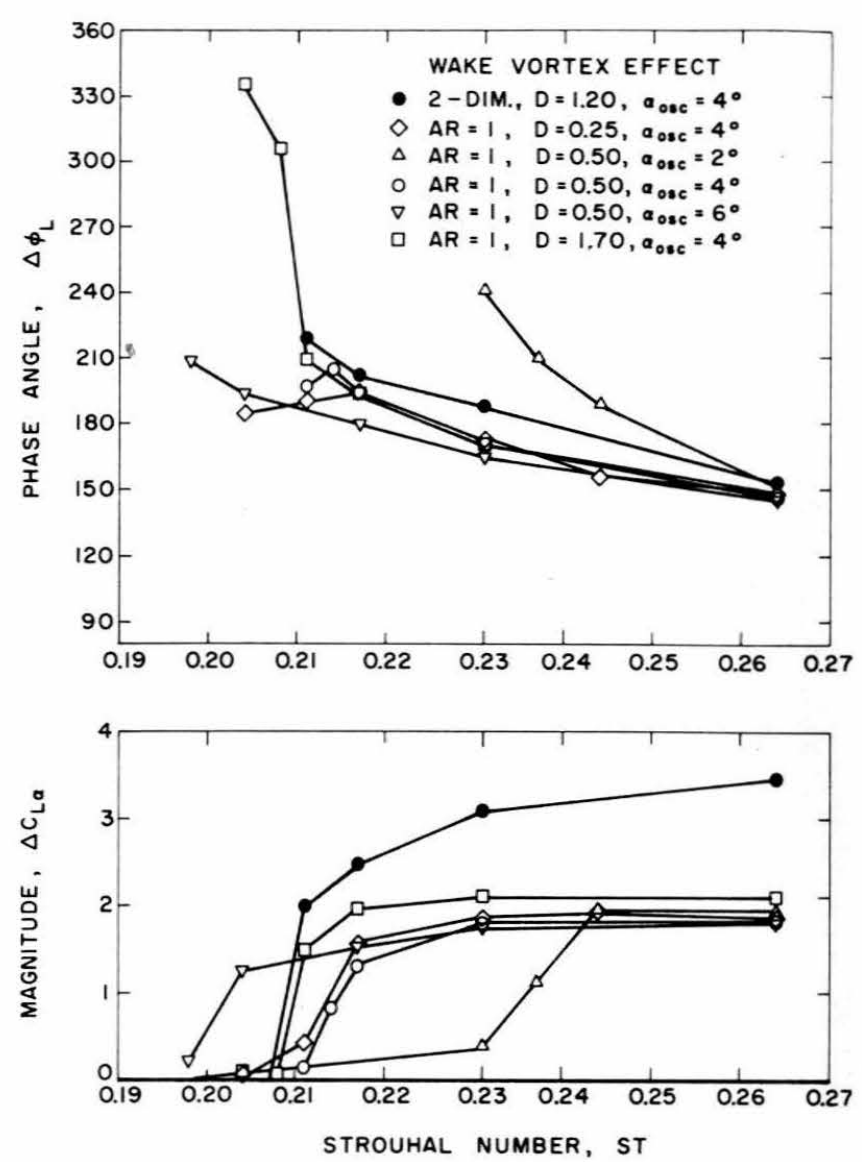

Fig. 17 "Wake vortex effect" plotted against Strouhal number for wedge foil at various values of submergence and oscillation amplitude

ascribed it to a deficiency in the amount of noncirculatory lift or apparent mass. On the basis of oscillation tests in still water, they showed the apparent mass was only 84 percent of its theoretical value.

It is not clear that the explanation is so simple, either for those tests or for the present ones. The tests of reference [17] varied the reduced frequency by changing only the flow velocity at a single constant oscillation frequency of $15 \mathrm{cps}$. In the present experiments, this point was further explored by conducting oscillation tests in still water over the range of test frequencies. From these, the measured apparent mass was found to decrease from 101 percent of the theoretical value for an infinite fluid at $3 \mathrm{cps}$ fairly smoothly to 80 percent at $40 \mathrm{cps}$, reaching, incidentally, the same 84 percent at $15 \mathrm{cps}$ as reported in [17]. The quantitative significance of these comparisons should not be overemphasized since the present tests were conducted in heave in a supercritical open-channel flow, while those of [17] were for pitching motion in a closed tunnel. As noted by Grace and Statler [18], in free-surface flows the separation of the total forces into the usual quasi-steady, wake-induced, and apparent-mass contributions is no longer such a use- 


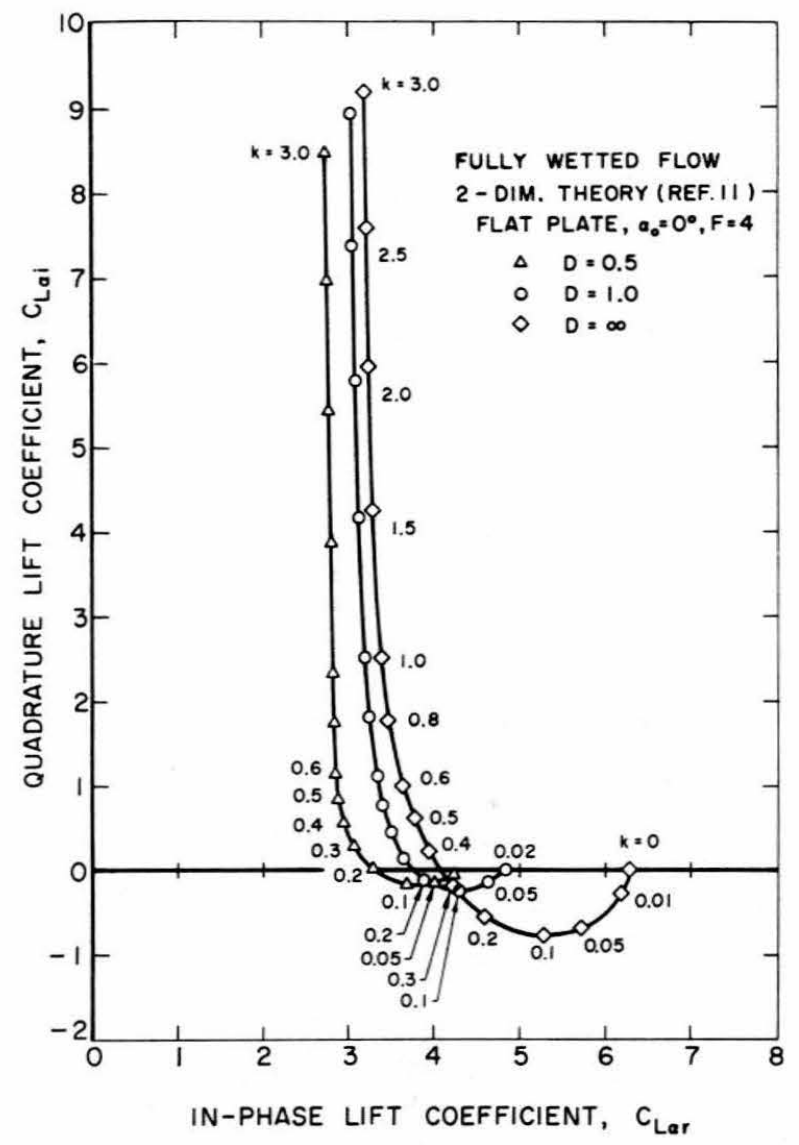

Fig. 18 Theoretical variation of unsteady lift coefficients with reduced frequency for a two-dimensional fully wetted flat plate, showing effect of submergence according to reference [11]

ful concept because of the interactions with the freesurface waves.

The simulation of two-dimensional flow, as in the present tests, by a finite-span model supported between walls is inevitably imperfect. The gap clearance between the model and the walls reduces the lift, as does the boundary layer along the walls. There is also some flow interference due to the strut supporting the hydrofoil. In addition, in the present tests there was some "breathing" of the two-dimensional inserts even after they had been stiffened; the gap which was normally set at 0.030 to $0.040 \mathrm{in}$. fluctuated by perhaps $0.010 \mathrm{in}$. at the lower frequencies. No fluctuations were observed at the higher frequencies. In steady flow a gap of this order would decrease the lift coefficient of a 6 -in. span foil by about 8 percent [16]. In the present oscillating tests, a quantitative estimate of this breathing effect would be hard to establish; however, summary estimates of the resulting fluctuations in flow incidence indicate an error of less than two percent due to this effect.

It should be pointed out that the calculations of reference [11] are for a fluid of infinite depth, whereas the present experiments, as mentioned, are for supercritical channel flow, theoretical computations for which do not
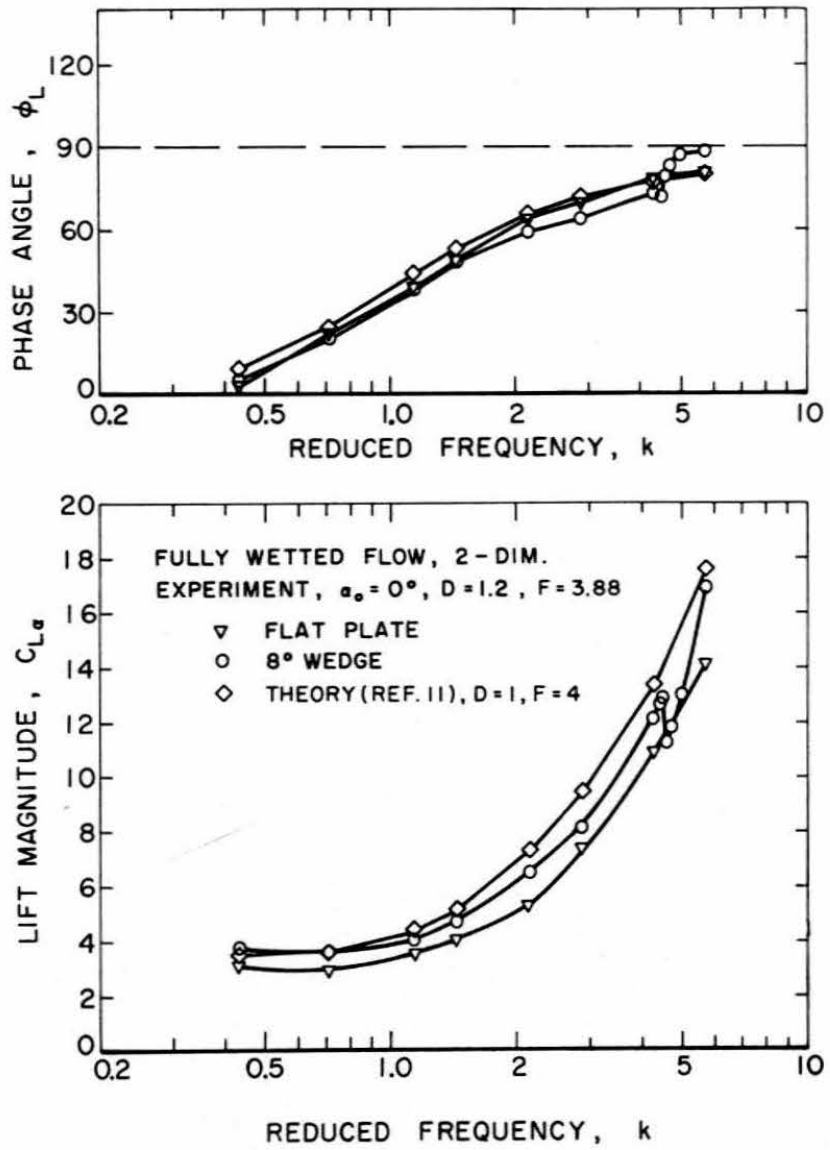

Fig. 19 Magnitude-and-phase plot of variation of unsteady lift coefficients with reduced frequency for two-dimensional foils, and comparison with the theory of reference [11]

appear to be available. The most comparable experimental work is that reported by Cieslowski and Pattison $[4,5]$, who performed pitching and heaving tests using a towing tank. Some pertinent results from [5] have been plotted in Fig. 16. The quadrature part of their measured lift is slightly less than the present one, both being rather less than the theoretical value. The principal difference between their results and those of the present work is in the in-phase lift, which is somewhat less in their measurements than found here at low reduced frequencies, the difference increasing notably with frequency.

Reduced Frequency. Tests at the higher flow velocity were also performed in two-dimensional flow for both the flat plate and the wedge foil. As in the aspect-ratio-one experiments, only small differences in the coefficients were observed. The differences to be expected because of the higher Froude number [11] are generally less than three percent.

\section{Base-Ventilated Flow}

Ventilation of the base of the wedge should have no measurable effect on the lift forces, since the flow about the foil itself is not materially changed; only one check 
run was made to confirm this supposition with the aspectratio-one wedge. These results, together with those for fully wetted foils at the same test conditions, are shown in Fig. 7. It can be seen that there are only minor differences between the lift coefficients for the baseventilated and the fully wetted wedge for reduced frequencies below the knee. For frequencies above the knee, the lift curve continues on a smooth path similar to that of the flat plate, bearing out that the vortex effect on the wedge is suppressed by base ventilation.

\section{Accuracy}

The statement of an overall accuracy is always problematical in experimental programs. In the present case, it was found that the short-term repeatability for runs separated by one or two weeks was within two percent, and results from the main data runs reproduced results from initial test runs of almost a year earlier within at worst five percent. Taking into account the other sources of error such as component calibrations and drift, and fluctuations in the physical parameters of the tests, the overall accuracy of the numerical results obtained is considered to be between five and ten percent. Usually, when only one parameter was varied, the measurements were carried out consecutively. Comparative differences between such tests do not involve more than the two-percent short-term uncertainty.

\section{Acknowledgments}

This work was supported under Department of the Navy Contract No. N00014-67-A-0094-0003. Research was carried out under the General Hydromechanics Research Program, Subproject SR 00901 01, administered by the David Taylor Model Basin for the Naval Ship Systems Command. We should like to thank Mr. T. Kiceniuk for his many helpful experimental suggestions and the staff of the Hydrodynamics Laboratory for their support.

\section{References}

1 Fourth Symposium on Naval Hydrodynamics, ACR-92, 0-735-602, Office of Naval Research, Washington, D. C., 1962.

2 H. Ashley, S. Widnall, and M. T. Landahl, "New Directions in Lifting Surface Theory," AIAA Journal, vol. 3, no. 1, January 1965 , pp. 3-16.

3 H. N. Abramson, W. H. Chu, and J. T. Irick, "Hydroelasticity," Southwest Research Institute, San Antonio, Texas, August 1966.

4 D. S. Cieslowski and J. H. Pattison, "Unsteady Hydrodynamic Loads and Flutter of Two-Dimensional Hydrofoils," SNAME Hydrofoil Symposium, 1965 Spring Meeting, Paper No. b.

5 J. H. Pattison, "Preliminary Revised Data for
(Reference 4)," private communication, November 1, 1966.

6 R. T. Knapp, J. Levy, J. P. O'Neill, and F. B. Brown, "The Hydrodynamics Laboratory of the California Institute of Technology," Trans. ASME, vol. 70, July 1948 , pp. 437-457.

7 W. R. Laidlaw, "Theoretical and Experimental Pressure Distributions on Low Aspect Ratio Wings Oscillating in an Incompressible Flow," MIT Aeroelastic and Structural Research Laboratory Technical Report 51-2, September 1954.

8 I. H. Abbott and A. L. van Doenhoff, Theory of Wing Sections, Dover Publications, New York, 1959.

9 R. P. Bernicker, "A Linearized Two-Dimensional Theory for High-Speed Hydrofoils Near the Free Surface," Journal of Ship Research, vol. 10, March 1966, pp. $25-48$.

10 L. C. Woods, "The Lift and Moment Acting on a Thick Aerofoil in Unsteady Motion," Philosophical Transactions of the Royal Society of London, vol. 247, Series A, November 1954, p. 131.

11 P. Crimi and I. C. Statler, "Forces and Moments on an Oscillating Hydrofoil," Cornell Aeronautical Laboratory Report BB-1629-S-1, August 1962; also see Fourth Symposium on Naval Hydrodynamics, 1962, pp. 477-494.

12 K. L. Wadlin and K. W. Christopher, "A Method for Calculation of Hydrodynamic Lift for Submerged and Planing Rectangular Lift Surfaces," NACA TN 4168, 1958.

13 J. O. Young and J. W. Holl, "Effects of Cavitation on Periodic Wakes Behind Symmetric Wedges," ASME Paper No. 65-FE-15.

14 R. E. D. Bishop and A. Y. Hassan, "The Lift and Drag Forces On a Circular Cylinder in a Flowing Fluid," Proceedings of the Royal Society of London, vol. 277, Series A, 1964, pp. 32-75.

15 P. S. Eagleson, J. W. Daily, and G. K. Noutsopolous, "Flow-Induced Vibration of Flat Plates: The Mechanism of Self-Excitation," MIT Hydrodynamics Laboratory Report No. 58, February 1963.

16 R. W. Kermeen, "Water Tunnel Tests of NACA 4412 and Walchner Profile 7 Hydrofoils in Non-Cavitating and Cavitating Flows," California Institute of Technology Hydrodynamics Laboratory Report No. 47-5, February 1956.

17 F. E. Smith and M. Sevik, "An Investigation of the Forces on an Airfoil Oscillating in Pitch About the Quarter Chord," Pennsylvania State University, Ordnance Research Laboratory TM 607.2441-02, March 1964.

18 J. M. Grace and I. C. Statler, "Free-Surface Effects on the Apparent-Mass, Quasi-Steady, and Wake Contributions to Hydrofoil Loads," Cornell Aeronautical Laboratory Report No. BB-1629-S-2, August 1965. 